Ann. Zootech., I96r, 10 (2), II3 - I2.5.

\title{
RELATIONS ENTRE LA QUANTITÉ DE LAIT CONSOMMÉ PAR LES AGNEAUX ET LEUR CROISSANCE
}

\author{
G. RICORDFAU et R. BOCCARD. \\ Avec la collaboration technique de C. Dayiasi et A. Van Wit.Ligen \\ Station de Recherches sur lifleziage, \\ Centre national de Recherches zontechniques, Jouy-en-Josas (Seine-et-Oise).
}

\section{SOMMAIRE}

A partir des données obtenues sur 97 brebis de la race des Préalpes du Sud (.58 brebis allaitant I agneau, 39 allaitant des jumeaux ou bessons), nous avons analysé quelques facteurs de variation de la croissance des agneaux au cours des premières semaines d'allaitement : la cuantité de lait consommé et le poids des agneaux à la naissance.

Les résultats montrent:

$\mathrm{I}^{\mathrm{O}}$ Que la corrélation maximum entre lait tété et croissance est atteinte plus tôt pour les agneaux bessons (période o-21 jours) que pour les agneaux simples (o-35 jours).

$2^{0}$ Que le poids des agneaux entre 29 et 35 jours pernet une aussi bonne est imat ion de la quantité de lait consommé pendant la période correspondante que la connaissance de la vitesse moyenne de croissance et du poids à la naissance.

$3^{\circ}$ Que dans tous les cas, l'écart-type des différences entre quantité rédlement consommée et quantité estimée est de l'ordre de i 2 p. Ioo.

$4^{\circ}$ Que pendant les 5 premières semaines, la vitesse de croissance des agneaux simples ne dépend pas intrinsèquement de leur poids de naissance, comme cela semble être le cas pour les jumeaux. et les bessons.

$5^{\circ}$ Que l'indice de consommation est respectivenent de 5,84 ct 5,4 I pour les agneaux simples

Pour la majorité des brebis laitières soumises à la traite, la durée d'allaitement s'étale sur une période de 40 à 45 jours. I) point de vue théorique la méthodologie du contrôle laitier impose donc la connaissance précise de la production laitière des brebis au cours de cette période. Il ne nous appartient pas dans cette étude, d'en analyser tous les aspects, dont le plus important est celui de la relation avec 1'aptitude laitière des brebis à la traite, mais de voir dans quelle mesure il est possible d'utiliser le gain de poids des agneaux ou, plus simplement, leur poids vif comme estimateur du lait tété par les agneaux.

RITZMAN (I9I7), NEIDIG et IDDINGS (I9I9) ont été parmi les premiers à étudier l'influence de la production laitière des brebis sur la croissance des agneaux. 


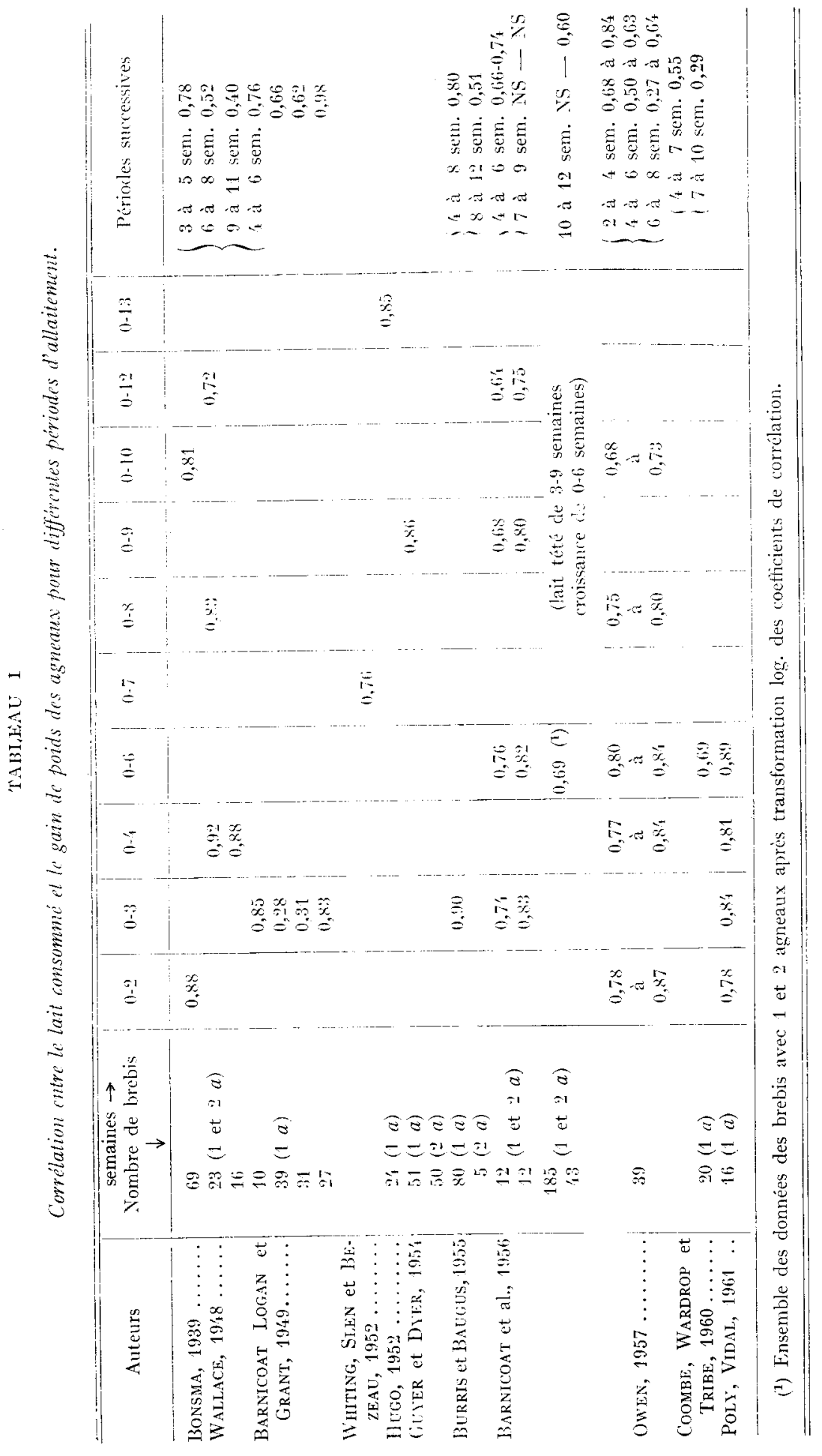


LEROY (1936), puis LEROY, IARY, ZELTER (I952) ont analy'sé et discuté les bases du contrôle laitier indirect à partir de la croissance des jeunes, en tenant compte de la composition du lait et de l'âge des agneaux.

Le tableau I présente les coefficients de corrélation obtenus par différents auteurs entre le lait consommé et la croissance des agneaux. La corrélation varie avec la période d'allaitement considérée. 'Tout en restant toujours élevée, elle est maximum pour la période de l'allaitement exclusif, c'est-à-dire le premier mois de lactation.

La connaissance de la composition du lait, en matière grasse notamment, est susceptible à priori de rendre plus étroite cette relation. Les coefficients de corrélation obtenus par BARNICOAT et al. (1956) entre la croissance enregistrée de o à I 2 semaines, les quantités de matière grasse d'une part, et de lait d'autre part (quantités consommées entre 3 et 9 semaines), ne sont pas significativement différents, bien que le premier soit plus élevé $(0,72$ contre $0,6 \mathrm{I})$.

Ritzman (I9I7), Niidig et Indings (I9I9), et Kovac (I955) arrivent d'ailleurs à la conclusion que la richesse du lait en matière grasse n'influe pas de façon significative sur la vitesse de croissance des agneaux. En fait, il faudrait peut-être tenir compte, non seulement des quantités de matières azotées ou de matière grasse ingérées, mais aussi de la richesse moyenne en ces éléments an cours des différentes périodes de croissance. Par exemple, dans l'espèce bovine, Mathreu ( Ig60) a observé que jusqu'à un mois la vitesse de croissance des jeunes veaux, recevant la même quantité de lait, mais à différentes teneurs en matière grasse, présentait une valeur maximum pour un taux butyrenx voisin de $35 \mathrm{~g}$. par litre. Selon le même autenr, ce taux optimum augmente avec l'âge des veaux. Si les mêmes phénomènes se produisent chez l'agneau, il serait nécessaire d'effectuer l'étude de la phase d'allaitement par périodes plus courtes que celles qui ont été envisagées jusqu'alors, notamment par BARNICOAT et al. (I956).

De telles études sont d'ailleurs délicates, car il est très difficile d'obtenir par la traite un échantillon représentatif du lait consommé par les agneatux. En effectuant 2 ou 3 traites pendant l'allaitement sur une période de 24 heures après séparation des agneaux, Constantinescu et Gondos (I958), Barnicoat et al. (I956), RicorDEAU et I)ENAMUR (I96I) ont montré que ces traites ne permettaient d'obtenir que 70 à 85 p. Ioo du lait consommé par les agneaux. Pour toutes ces raisons, nous n'avons pas tenu compte dans cette étude de la teneur en matière grasse du lait tété.

\section{MATÉRIEI ET MÉTHODE}

lin I957. 58 et 59, nous avons estimé la quantité de lait tété, entre la naissance et le sevrage, par les agneaux de la race des Préalpes du Sud selon une technique discutée précédemment (RICORDEAU, BOCCARD, DENAMUR, I960). Nos observations ont porté sur 97 brebis dont 43 seulement ont allaité leurs agneaux au-dela de 5 semaines.

\section{RÉSUI,TATS}

a) Evolution de la relation lait tété - croissance au cours des 7 premières semaines d'allaitement:

Pour les deux types d'agneaux (simples et bessons, tableau 2), la corrélation maximum $r_{12}$ (entre lait consommé et croissance) est comprise entre 0,75 et 0,80 . 
Cette valeur est atteinte plus tôt pour les agneaux bessons (période o-2I jours) que pour les agneaux simples (o-35 jours). Ce décalage résulte certainement de l'évolution différente de la production laitière des brebis allaitant soit un agneau, soit deux agneaux, la production maximum journalière étant atteinte beaucoup plus tôt pour les bessonnières (RICORdEAU, DENAMUR, I96I).

TABLEAU 2

Relations entre quantité de lait tité (1), ritesse moyenne croissance (2), et poids les agneaux à la fin de la période considérée (3).

\begin{tabular}{|c|c|c|c|c|c|c|}
\hline \multirow{2}{*}{$\begin{array}{c}\text { l'ériode } \\
\text { de croissance }\end{array}$} & \multicolumn{2}{|c|}{25 agneaux simples } & \multicolumn{2}{|c|}{29 couples de bessons } & \multicolumn{2}{|c|}{ Ensemble (j't brebis) } \\
\hline & $r_{12}$ & $r_{13}$ & $\gamma_{12}$ & $r_{13}$ & $r_{12}$ & $\gamma_{13}$ \\
\hline $0-1 /{ }^{\prime} \mathrm{j} . \ldots$ & 0,315 & 0,535 & 0,636 & 0,617 & 0,718 & 0,793 \\
\hline $0-21 \mathrm{j}$. & $0,6: 3$ & $0,68:=$ & 0,768 & 0,706 & 0,835 & 0,865 \\
\hline $0.28 \mathrm{j} . \ldots$ & 0,700 & 0,708 & 0,713 & $0,65^{\prime} t$ & 0,812 & 0,855 \\
\hline $0-35 \mathrm{j} . \ldots \ldots \ldots$ & 0,751 & 0,777 & 0,750 & 0,753 & 0,871 & 0,859 \\
\hline $0-42$ j. $\ldots$ & $0,73: 3$ & 0,765 & 0,682 & $0,52{ }_{x}^{\prime}$ & 0,851 & 0,852 \\
\hline $0-49 \mathrm{j} . \ldots \ldots \ldots \ldots$ & 0,733 & $0,7 \%$ & - & - & - & - \\
\hline
\end{tabular}

L'examen dı tableau 2 nous permet de faire deux remarques supplémentaires :

- le remplacement de la vitesse moyenne de croissance par le poids vif améliore presque toujouts le coefficient de détermination de la quantité de lait consommé ;

- le groupement des données relatives aux deux types d'agneaux conduit à une surestimation des corrélations $\gamma_{12}$ et $\gamma_{13}$ (entre lait tété et poids vif).

b) Période de référince: les 5 premières somaines d'allaitement:

Nous disposons pour cette période des domnées concernant $5^{8}$ agneaux simples et 39 couples de bessons (tablean 3).

TABLEAU 3

Relations culre la quantiti de lait consommí, la intesse moyenne de croissance et le poids des agneaux (5 premières semaines d'allaitement).

\begin{tabular}{|c|c|c|c|}
\hline Éléments considérés & $\begin{array}{l}\text { Corrélation, éart-type lié } \\
\text { et équation cle regression }\end{array}$ & 58 agneaux simples & 39 couples de bessons \\
\hline $\begin{array}{l}\text { Lait tété }(1) \text { et vitesse de } \\
\text { croissance }\left({ }_{2}\right) \ldots \ldots \ldots \ldots\end{array}$ & $\begin{array}{c}r_{12} \\
\sigma_{1,2}(\mathrm{~g}) \\
\sigma_{1,2}(\mathrm{p} \cdot 100) \\
\text { Equation de régression }\end{array}$ & $\begin{array}{c}0,702 \\
189 \\
13,0 \\
y_{1}=4,59 x_{2}+311\end{array}$ & $\begin{array}{c}0,751 \\
221 \\
11,2 \\
y_{1}=3,50 x_{2}+680\end{array}$ \\
\hline $\begin{array}{l}\text { Lait tété }(1) \text { et poids vif } \\
\text { moyen entre } 29 \text { et } 35 \mathrm{j}(3)\end{array}$ & $\begin{array}{c}r_{13} \\
\sigma_{1,3}(g) \\
\sigma_{1,3}(\mathrm{p} .100) \\
\text { Eqquation de régression }\end{array}$ & $\begin{array}{c}0,717 \\
185 \\
12,7 \\
y_{1}=3,43 x_{3}+1 \mathbf{1}^{\prime} 4\end{array}$ & $\begin{array}{c}0,753 \\
220 \\
11,2 \\
y_{1}=2.79 x_{3} \div 369\end{array}$ \\
\hline
\end{tabular}

A un mois, l'écart-type des erreurs entre la quantité journalière de lait réellement consommée et celle estimée à partir de la vitesse de croissance et du poids vif, est de l'ordre de $\mathrm{I} g \mathrm{o} g$ pour les brebis avec un agneau et de $220 \mathrm{~g}$ pour les bessonnières, soit I I à $\mathrm{I} 3 \mathrm{p}$. Ioo en valeur relative. Les coefficients de régression calculés à partir 
des données des brebis avec I et 2 agneaux ne sont pas significativement différents $\left(\mathrm{P}=0, \mathrm{I} 7\right.$ et 0,29 pour la comparaison des coefficients de régression $b_{12}$ et $\left.b_{\mathbf{1 3}}\right)$. Toutefois, la régression du lait tété sur la vitesse de croissance ou le poids vif est plus faible pour les bessons que pour les simples.

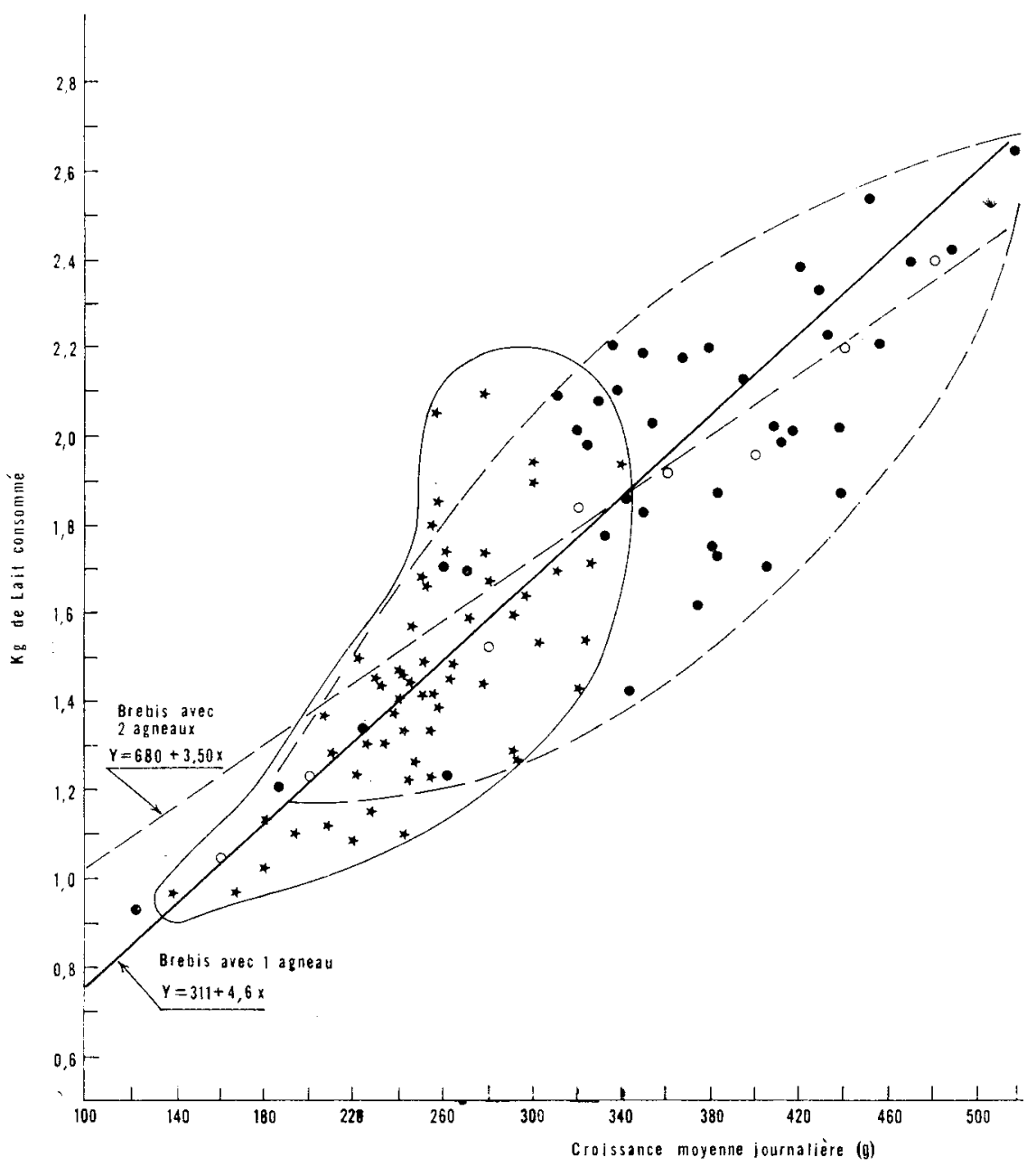

HiG. I. - Relation entre la croissance des agnearx et la quantité de lait consommée de 0 à 32 jours en noyenne.

* agneaux nés simples

- agneaux jumeaux fimites de la distribution.

- Moyenne réelle de lait consommé par classe de vitesse de croissance.

WALLACE (I948) a observé d'ailleurs des résultats comparables. A partir de données provenant de $I 6$ brebis Suffolk (allaitant en majorité des simples) et de 23 brebis Border Leicester $\mathrm{x}$ Cheviot (allaitant en majorité des bessons), il constate une corrélation de 0,902 entre croissance et lait consommé $\left(r_{12}\right)$, contre une corrélation de 
0,915 lorsqu'on considère le poids vif à 28 jours au lieu de la vitesse de croissance $\left(r_{13}\right)$. D'autre part, la régression $b_{13}$ de la quantité de lait consommé sur le poids vif à 28 jours est supérieure pour les brebis Suffolk $(3,395)$ à celle des brebis Border Leicester $\mathrm{x}$ Cheviot $(3,305)$.

Nous avons admis 1'hypothèse d'une régression linéaire entre le lait consommé et la vitesse moyenne de croissance. En fait, il semble plutôt que la ligne de régression soit curviligne, notamment en ce qui concerne les agneaux simples ayant une vitesse moyenne de croissance journalière supérieure à $25^{\circ} \mathrm{g}$. Si nos données sont insuffisantes pour tester la linéarité de la régression, on peut constater d'une part que les agneaux qui consomment peu de lait semblent l'utiliser proportionnellement mieux pour leur croissance, bien que, d'autre part, il existe une relation légèrement négative entre la vitesse de croissance et la quantité de lait nécessaire par unité de croît.

Ces deux conclusions, apparemment divergentes, ne peuvent s'expliquer que si l'on place le problème sur le plan énergétique en faisant la part des besoins d'entretien et de croissance. On a bien mis en évidence, par exemple, que la quantité de matière grasse fixée par l'organisme est d'autant plus élevée que la croissance est plus rapide :

TABLEAU 4

Composition corporelle de quelqu's agnean Prialpes.

\begin{tabular}{|c|c|c|c|c|c|c|c|c|}
\hline \multirow{2}{*}{$\begin{array}{c}\text { Poicls à la } \\
\text { naissance } \\
(\mathrm{kg} g)\end{array}$} & \multicolumn{2}{|c|}{ Abattage } & \multirow{2}{*}{$\begin{array}{c}\text { Vitesse de } \\
\text { croissance } \\
\text { (en g jar } \\
\text { jour) }\end{array}$} & \multirow{2}{*}{$\begin{array}{c}\text { Poids des } \\
\text { rarcasses } \\
\text { (kg) }\end{array}$} & \multicolumn{3}{|c|}{$\begin{array}{c}\text { Poids des tissus disséqués dans } \\
\text { une demi-carcasse (g) }\end{array}$} & \multirow{2}{*}{$\begin{array}{l}\text { Tissus adi } \\
\text { peux dissé } \\
\text { quér dans } \\
\text { 'arcasse } \\
\text { en } \quad \text { |. } 100\end{array}$} \\
\hline & $\begin{array}{l}\text { Poids vif } \\
\text { (kg) }\end{array}$ & $\begin{array}{l}\text { dige } \\
\text { (jour) }\end{array}$ & & & osseux & musculaires & adipenx & \\
\hline 4,3 & 5,6 & 13 & 100 & 2,7 & 360 & 822 & 26 & 2.0 \\
\hline$\{, \underline{\prime}$ & 7,4 & 13 & 当借 & 3,9 & 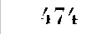 & 1222 & $10: 3$ & $\therefore, 2$ \\
\hline $1,1, \ldots$ & 7,6 & $1 / 4$ & 253 & 1,1 & 463 & $1: 98$ & 105 & $\therefore, 1$ \\
\hline $3,6 i$ & 4,2 & 25 & $\ddot{4}$ & 1 , & 2660 & 476 & néant & 0 \\
\hline 3,6 & 9,5 & 30 & $1 \times 6$ & 4 & $5 x$ & 154 & 66 & 2.7 \\
\hline 4,5 & 10,6 & 26 & $2: 3$ & 6 & bi2 & 1952 & 182 & $(i, 0)$ \\
\hline $4,3 \ldots \ldots$ & 12,2 & 29 & $27: 2$ & $6, i^{\prime}$ & 607 & 1916 & $3: 9$ & 111,5 \\
\hline
\end{tabular}

le tableau 4, donnant la composition corporelle en tissus dissécables d'agneaux de croissances et d'âges différents, illustre bien cette évolution pour le cas précis des agneaux de la race des Préalpes (BOCCARD, I960). Il en résulte que l'énergie nécessaire par unité de gain de poids vif augmente non seulement avec l'âge, mais avec la vitesse de croissance, comme l'ont montré LFROY et ZELTER (I948). Les animaux à croissance rapide auraient donc, pour la transformation des nutriments en gain de poids, un rendement énergétique plus faible que les animaux à croissance plus lente. Guyer et Dyer (I954), Munro (I955), Hunter (I956) et Owen (I957) sont arrivés aux mêmes conclusions. Cependant, cette diminution du rendement énergétique est compensée par la valeur plus forte du rapport de l'énergie consacrée à la croissance à celle utilisée pour l'entretien pendant une même période. D'autre part, l'énergie nécessaire à l'entretien d'une unité de poids vif décroît avec l'accroissement du poids vif de l'animal. En définitive, pour atteindre un poids vif déterminé, 
sans considération de composition corporelle, les agneaux à croissance rapide ont un rendement énergétique global supérieur à ceux qui ont une croissance lente. Cet aspect a d'ailleurs été analysé par WALLACE (I955).

c) Indice de consommation:

La quantité de lait nécessaire par unité de gain de poids vif ou indice de consommation a été calculée par de nombreux auteurs pour des races et des périodes de contrôle différentes. Les valeurs moyennes obtenues (tableau 5), pour la période des 6 premières semaines d'allaitement varient de 4 à 6 , et peuvent même atteindre des valeurs supérieures si l'on se réfère aux estimations de Munko (I955) obtenues à partir des données de Neiding et IDDings, Bonsma, BARnicoAT et al. Il existe

TABLEAU 5

Indices de consonmation des agneaux de différentes races pour les premicres semaines de croissance.

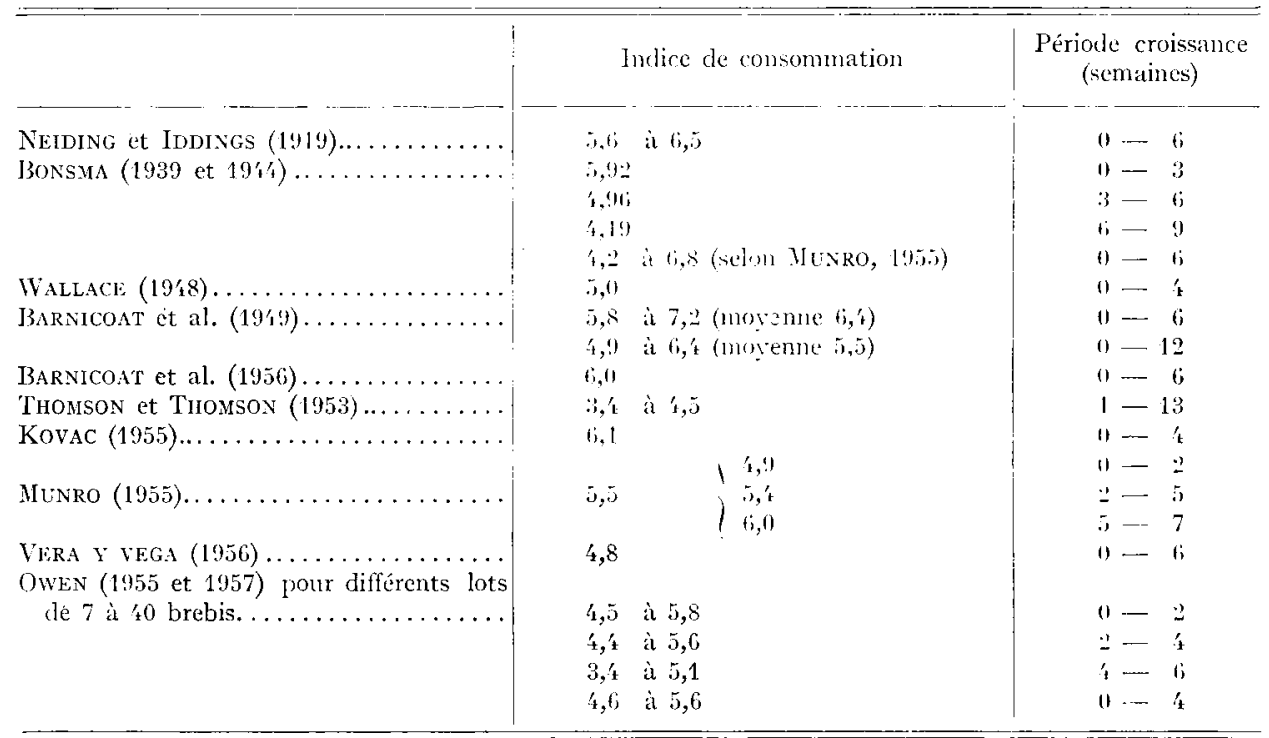

donc des différences individuelles, raciales et annuelles très importantes, ce qui n'est pas très étonnant si l'on tient compte de la grande variabilité des aptitudes laitières et du poids des agneaux à un âge donné. En moyenne, l'indice est plus faible pour les mâles, mais la différence n'est pas significative selon VíRA Y VEGA (I956). Ce dernier constate également, comme OWEN (I957), une relation légèrement positive entre le niveau de production laitière des brebis et l'indice de consommation. En ce qui concerne les agneaux Préalpes simples et bessons, les indices relatifs aux 5 premières semaines d'allaitement sont respectivement de $5,8_{4}$ et $5,4 \mathrm{I}$ (tableau 6 ), avec un coefficient de variabilité de I3,6 p. Ioo.

Sans vouloir discuter le point de vue énergétique, pour les raisons déjà énoncées et d'autant plus que nous ne pouvons considérer chaque agneau isolément, il est intéressant d'entegistrer la différence moyenne observée entre agneaux simples et 
agneaux bessons. A un mois, par exemple, le couple de bessons n'a consommé que 35 p. roo de lait de plus que les simples, alors que sa croissance a été supérieure de 44 p. Ioo. En admettant que la composition du lait des bessonnières ne soit pas dif-

TABLEAU 6

Indice de consommation des agneaux Préalpes.

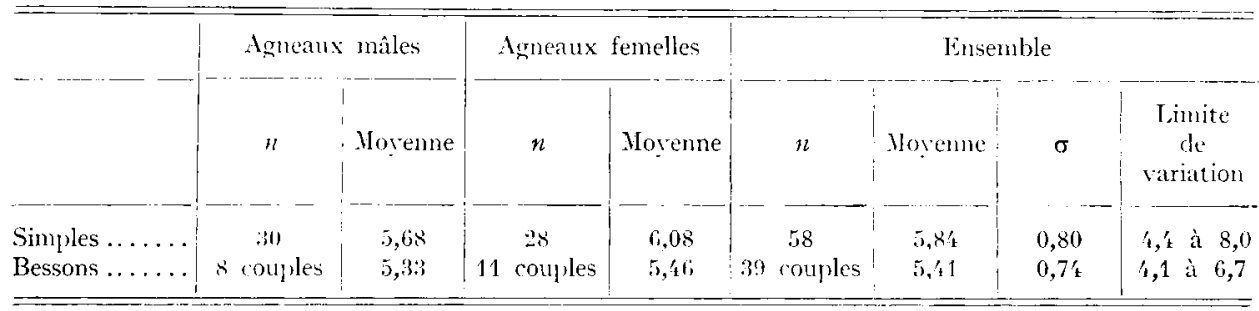

férente de celle des brebis allaitant un seul agneau, plusieurs hypothèses peuvent expliquer cette supériorité :

Io Les bessons pris individuellement sont, à un âge donné, plus légers que les agneaux simples. Ils peuvent, par conséquent, consacrer à leur croissance une part de lait proportionnellement plus importante que ne peuvent le faire les agneaux simples.

$2^{\circ}$ La vitesse de croissance des bessons à l'allaitement est toujours plus faible que celle des simples qui forment plus de tissus adipeux.

$3^{\circ}$ Les bessons tètent proportionnellement plus de lait que les simples au début de la période d'allaitement. Dans une étude sur la production laitière des truies, SALMON LEGAGNEUR ( $195^{8}$ ) signale, en effet, que la répartition de la production laitière a une influence sur la croissance des porcelets : les truies qui ont la plus forte production au début de la lactation étant celles qui produisent les portées les plus lourdes.

\section{d) Influence du poids ì la naissance:}

Le poids de naissance des agneaux, dans la mesure où il traduit la vigueur et l'état de l'agneau au début de la phase d'allaitement, est susceptible d'influencer la relation lait consommé-gain de poids. Là aussi, nous séparerons les deux types d'agneaux pour tenir compte des différences qui peuvent exister dans leur développement et mettre ainsi en évidence les interactions existantes.

I $^{\circ}$ Cas des agneaux simples. - Les corrélations totales $r_{14}$ et $r_{24}$ entre le poids à la naissance d'une part (4), la production laitière des mères (I) et la vitesse moyenne de croissance (2) des 58 agneaux d'autre part, sont peu significatives (tableau $7 \mathrm{a}$ ). Par contre, les corrélations partielles $r_{14 \cdot 2}$ et $r_{21 \cdot 1}$ ne sont plus significatives. Ces résultats montrent que :

- pour des agneaux ayant la même vitesse moyenne de croissance, la quantité de lait consommé est indépendante du poids de naissance des agneaux;

- la vitesse de croissance des agneaux simples ne dépend pratiquement pas de leurs poids à la naissance, mais presque essentiellement de la quantité de lait consommé. 


\section{TABLEAU 7}

Relations entre la production laitière des mères et la croissance de leurs agneaux indépendamment ou en fonction du poids des mères à la mise-bas et du poids de naissance des agneaux

a) 58 agneaux simples

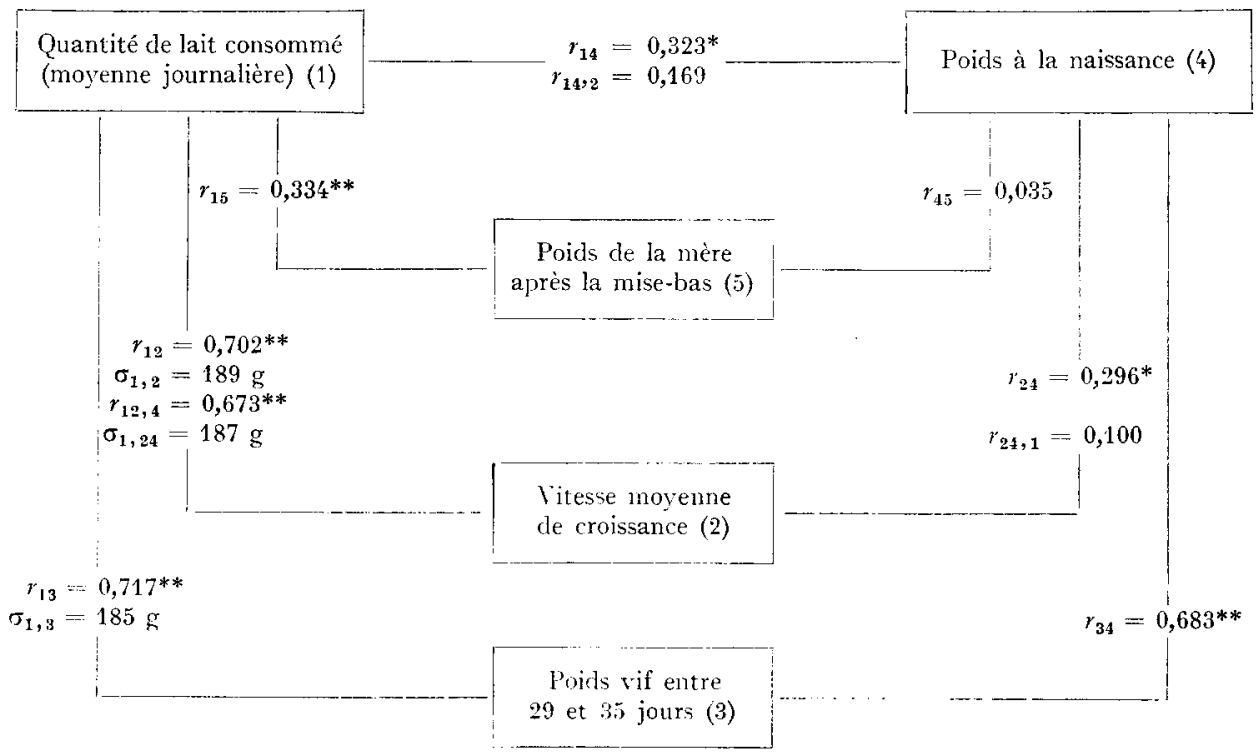

b) 39 couples de jumeaux

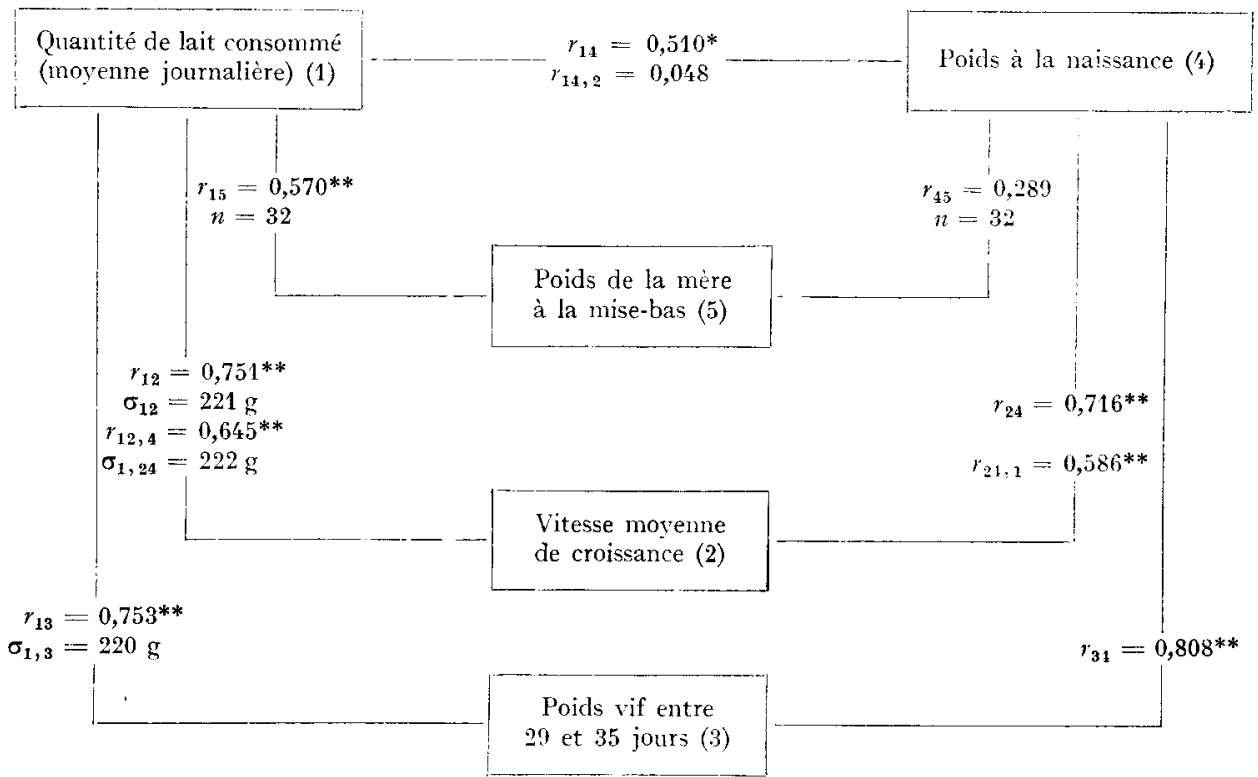

$\begin{array}{cccc}\text { agneaux simples } & \text { agneaux jumeaux } \\ \text { moyenne } & \sigma & \text { moyenne } & \sigma \\ - & - & - & - \\ 1445 & 266 & 1970 & 335 \\ 256 & 41 & 370 & 72 \\ 4,1 & 0,8 & 6,4 & 0,8\end{array}$


Les corrélations $r_{12}$ et $r_{12,4}$ sont respectivement de 0,70 et 0,67 , mais l'écarttype doublement lié $\sigma_{1,24}$ (c'est-à-dire l'écart-type de la distribution des quantités de lait consommées d'une sous-poptulation obtenue en sélectionnant d'abord les agneaux de même poids à la naissance, puis, parmi ceux-ci, les agneaux ayant la même vitesse de croissance )est équivalent à l'écart-type lié $\sigma_{1,2}\left(\sigma_{1 \cdot 24}=\mathrm{r} 88 \mathrm{~g}.\right)$.

In ce qui concerne la corrélation partielle entre lait consommé jusqu'à 9 semaines et la vitesse moyenne de croissance correspondante (le nombre d'agneaux qui tètent et le poids à la naissance étant maintenus constants), GUYER et I) YER (I954) obtiennent une valeur de 0,690 alors que la corrélation totale observée $\left(r_{12}\right)$ était de 0,856 pour l'ensemble des observations. Pour ces auteurs, si l'on tient compte de la production laitière des mères, le poids de naissance des agneaux n'influence pas le taux de croissance $\left(r_{21,1}=0, \operatorname{Io} 8, \mathrm{~N}\right.$. S. contre $\left.r_{2,4}=0,649\right)$. A partir de 40 brebis, BARnicoat et a1. (r956) rapportent une corrélation multiple $\left(R_{2.4}\right)$ de 0,735 entre la vitesse moyenne de croissance des agneaux, d'une part, et l'ensemble lait consommé et poids à la naissance d'autre part, contre une corrélation totale $\left(r_{12}\right)$ de 0,73 I entre la vitesse moyenne de croissance et la quantité de lait tété. La considération du poids à la naissance dans la détermination du lait consommé par les agneaux simples ne permet done pas d'améliorer sensiblement la précision de l'estimation et 1'on peut penser, avec WAILACE (I948), que le poids vif à un mois est au moins un aussi bon estimateur de la quantité de lait consommé que la croissance des agneaux pendant la même période

$2^{\circ}$ Cas des agneaux doubles. - Les corrélations $r_{14}$ et $r_{24}$ sont très significatives et beaucoup plus élevées que dans le cas des agneaux simples, mais alors que la corrélation partielle $\gamma_{14,2}$ est pratiquement nulle, la corrélation $r_{24,1}$ reste significative. Pour une même consommation de lait, la vitesse de croissance des agneaux augmenterait donc avec le poids de naissance du couple de bessons.

Le problème ne doit d'ailleurs pas être aussi simple et l'on peut supposer (nos données étant insuffisantes pour le confirmer) qu'indépendamment de la quantité de lait consommé, la relation poids à la naissance - vitesse moyenne de croissance n'est pas linéaire. On aurait ainsi le schéma suivant:

- une limite inférieure du poids à la naissance en dessous de laquelle le rendement lait-gain de poids est médiocre,

- une limite supérieure du poids à la naissance au-dessus de laquelle la vitesse de croissance ne dépend pas du poids à la naissance (cas de la majorité des simples),

- une classe intermédiaire oì se manifeste la croissance compensatrice (cas de la moyenne des agneaux doubles). En effet, les fœtus d'une brebis bessonnière bénéficient de moins bonnes conditions in utero que le fœtus unique dont la croissance prénatale n'est pas freinée, grâce à des conditions de milieu plus favorables. Après la naissance, les bessons se trouvent placés dans des conditions de milieu meilleures qui leur permettent une croissance plus rapide. Ce phénomène, tel qu'il a été étudié chez les bovins (WINCHESTER et Howe, I955), suppose des conditions de vie sousoptimales, pendant une certaine phase de la croissance, pour pouvoir se manifester par la suite. Ce point particulier n'a été que peu étudié chez les ovins, les auteurs ne séparant généralement pas les données des agneaux simples et des agneaux bessons. GUYER et DYER (I954) ont cependant observé une corrélation partielle, non significative, entre le poids de naissance et la croissance, la quantité de lait tété et le nombre d'agneaux par brebis allaitante étant maintenus constants, mais leur période de référence est celle des 63 premiers jours. 


\section{3 - Ensemble des agneaux:}

Comme nous l'avons déjà signalé, les différences moyennes entre agneaux simples et bessons résultent du fait que pour un âge donné les deux catégories d'agneaux n'ont pas le même poids. L'analyse de la covariance portant sur la comparaison des vitesses de croissance des deux catégories d'agneaux ayant la même consommation de lait et le même poids à la naissance, nous permet cependant de conclure que les hypothèses de plans de régression parallèles pour les deux catégories et de plans de régression confondus qu'ils soient ot1 non supposés parallèles sont très acceptables.

Par la même méthode nous avons pu constater :

- que pour un même poids de naissance et une même vitesse de croissance, les données ne permettent pas de mettre en évidence une différence significative de la quantité de lait consommée par les deux catégories;

-- que 1'influence des variations du poids à la naissance et de la croissance sur la quantité de lait tété n'étaient pas significativement différentes pour les agneaux simples et jumeaux. La quantité moyenne journalière de lait tété $\left(\mathrm{y}_{1}\right)$ étant estimée dans ce cas, à partir de la croissance moyenne journalière $\left(\mathrm{x}_{2}\right)$ et le poids de naissance $\left(\mathrm{x}_{4}\right)$ par la formule suivante:

$\mathrm{y}_{1}=425,66+3,72 \mathrm{x}_{2}+26, \mathrm{I} 7 \mathrm{x}_{4}$

-.- que l'on peut, sans perte de précision, négliger le poids de naissance et utiliser pour l'ensemble des agneaux la formule de régression du lait tété sur la croissance.

$\mathrm{y}_{1}=4,08 \mathrm{x}_{2}+449$

l'écart type résiduel $\sigma_{y_{1}, x_{2}}$ étant estimé à $206 \mathrm{~g}$.

Les 2 catégories d'agneaux se comportent donc de la même façon, ce qui permet à la rigueur de grouper l'ensemble de leurs données.

\section{CONCIUSION}

De l'ensemble des résultats exposés, il ressort que la relation lait tété-croissance est étroite, mais cependant assez variable entre lots et races (Tableau I - 3 ou 4 premières semaines). Les comparaisons sont cependant rendues délicates du fait que les auteurs :

$I^{\circ}$ indiquent 1'intensité de la liaison, sans préciser les erreurs dans l'estimation du lait consommé (variance liée du lait consommé par rapport au poids vif ou au gain de poids),

$2^{\circ}$ ne séparent pas toujours les données des agneaux simples et bessons.

Après Wallace (I940), Guyer et DYER (I954), Barnicoat et a1. (I956), nous sommes arrivés à la conclusion que la considération du poids à la naissance offre cependant un intérêt certain, car elle rend possible d'autres comparaisons et doit permettre de déceler certaines erreurs dans l'alimentation ou le comportement des agneaux. D'autre part, la pratique de croisements, soit entre races, soit entre souches de formats différents, est susceptible de nous offrir des échantillons exceptionnels pour lesquels les relations établies ci-dessus ne seront plus satisfaisantes.

Dans notre étude, nous n'avons retenu que le poids vif contrôlé entre 29 et 35 jours (le poids moyen étant ajusté d'après la croissance réalisée depuis la naissance). La considération de périodes partielles doit permettre également d’obtenir des résultats intéressants, mais elle nécessite alors un plus grand nombre de pesées. 
Avec nos données, la précision de l'estimation du lait consommé soit à partir du croît, soit à partir du poids vif, est assez faible : écart-type des erreurs de l'ordre de I2 p. IOo. Si ces estimations penvent fournir des renseignements précieux dans le cadre d'un contrôle laitier indirect, pour le triage des brebis suivant leur aptitude laitière, les contrôles d'alimentation ou de descendance, il semble difficile d'en généraliser l'emploi dans le cadre du contrôle laitier direct, d'autant plus que sur de grands troupeaux, doivent être importantes les erreurs résultant des agneaux voleurs, des abandons et des adoptions, des accidents pathologiques divers, de l'usage plus ou moins précoce du sevrage progressif.

Reçu en févier $196 \mathrm{r}$.

\section{SUMMARY}

RELATIONSHIP BETWEEN GROWTH OF LAMBS AND QUANTITY OF MILK CONSUMED.

From data obtained from 97 ewes of the Prealpes du Sud breed ( 58 ewes suckling single, 39 suckling twins), certain factors influencing the growth of lambs during the first weeks of suckling have been studied viz. the quantity of milk consumed and the weight of lambs at birth.

The results show :

I. - That the maximum correlation between milk suckled and growth is reached earlier for twin lambs (period $0-2$ I days) than for single lambs (0-35 days).

2. - That the weight of the lambs between 28 and 35 days makes it possible to give an equally good estimation of the quantity of milk consumed during the corresponding period, as the knowledge of the average rate of growth and of the weight at birth.

3. - That in all cases the standard deviation of the differences between the quantity actually consumed and the quantity estimated is about $12 \mathrm{p}$. Ioo.

4. - That during the first five weeks, the rate of growth of single lambs does not depend intrinsically on their birth weight, as seems to be the case for twins.

5. - That the feed efficiency is 5,84 and $5,4 \mathrm{I}$ for single and twin lambs, respectively.

\section{RÉFÉRENCES BIBLIOGRAPHIQUES}

Barnicoat C. R., Logan A. G., Grant A. I., i949. Milk secretion studies with New-Zealand Romney ewes. Part. I. J. agric, Sci., 39, 44-45.

Barnicoat C. R., Murray P. F., Roberts E. M., Wilson G. S., I956. Milk secretion studies with New-Zealand Romnev ewes. J. agric. Sci., 48, 9-35.

BocCARD R., ig6o. Résultats non publiés.

Bonsma F. N., I944. Milk production studies with sheep. Fmg in S. Afr., 19, 3rr-324, 328.

Bonsma F. N., I944. Influence of milk yield on the growth of lambs. Fmg in S. Afr., 19, 395-400.

Burris M. J., Baugus C. A., 1955. Milk consumption and growth of suckling lambs. J. Anim. Sci., 14, I86-I9I.

Constantinescu O., Gondos G., 1958. Controlul productiei de lapte la oi în timpul alaptarii mieillor. Anal. Inst. Cerc. Zooteh., $15: 459-474$.

J.B. CoOmbe, I. D. WARDRop, D. E. TRIBE, I960. A study of milk production of the grazing ewe, with emphasis on the expérimental technique employed. J. agric. Sci., 54, 353. 359.

Guyer P. Q., Dyer A. J., I954. Study of factors affecting sheep production. Missouri Agric. Exp. Stat. Res. Bull. 558 .

Hugo W. J., I952. The influence of feeding on the milk production of the Merino ewe. Fmg in $S$. Afr., $27,5 \circ 3$.

Hunter G. C., 1956. 'I he maternal influence on size in sheep. J. agric. Sci., 48, 30. 60.

Kovac V., 1955. The milk consumption of growing lambs. in Anim. Breed. Abstr., 25, I 7 r.

LEROY A. M., I 956 Application éventuelle des méthodes de contrôle laitier à la sélection des races ovines précoces. C. R. Acad. Agric. Fr., 22, 733-738.

Leroy A. M., Zelter S. Z., i948. Fxpériences d'abattage successifs avec des agneaux Ile de France et Texel. Ann. agron., Paris no 2.

Leroy A. M., Lery G., Zelter S. Z., I952. Contribution à l'étude de l'utilisation du lait par les jeunes animaux. Ann. Zootech. Fr., 1, 6r-77.

Mathied C. Communication personnelle.

Munro J., i955. Studies on the milk yields of Scottish Blackface ewes. J. agric. Sci., 46, I3I-I 36. 
NeIDING R. E., Indings E. J., IgIg. Quantity and composition of ewes'milk its relation to the growth of lambs. J. agric. Res., 17, 19-33.

OWEN J.B., I957. A study of the lactation and growth of Hill sheep in their native environment and under lowland conditions. J. agric. Sci., 48, $387-412$.

OWEN J. B., 1955. Milk production in sheep. Agricullure, l.ond., 62, I 10-r I 4.

Poly. J., VIDAL P. Communication personnclle.

Ricordeau G., Dennamur R., ig6i. Résultats non publiés.

Ricordeau G., Boccard R., Denantr R., ig6o. Mesure de lit production laitiere des brebis pendant la période d'allaitement. Ann. Zootech., Fr., 9, 97-г20.

RITZMAN E. G., I917. Ewes'milk its fat content and relation to the growth of lambs. J. agric. Res., 8, 29-36.

Salmon-Legagxerr E., I958. Observations sur la production laitice des truics. Ann. Zookch., Fr., $7,143-162$.

Thomson W., Trouson A. M., i953. Effect of diet on milk vield of the ewe and growth of her lamb. Brit. J. Niurit., $7,263-274$.

VERA Y VEGA A., I956. Comparacion de dos métodos de estimacion de la produccion lechera en la oveja clurante el periode de amamantamiento del Cordero. Arch. Zootec., 5, 3:2-348.

WALLACE L. R., I 948. Growth of lambs before and after birth in relation to the level of nutrition. Part. I. J. agric. Sci., 38, 93- 53 .

WHitrixg F., SEEEN S. B., BrzeAt M., I952. The quantity and quality of mature ewes'milk as influenced by level of protein in the ration. Sci. Agric., 32, 365-3it.

Wallace L. R., 1955. Factors influencing the efficiency of feed conversion by the sheeps. Proc. Brit. Nutrit. Soc., 14, 7-13.

Winchester C.F., Howe P. E., 1955. Relative effects of continuous and interrupted growth on beef steers. L.S. Dept. Agric. Techn. Bull. no I. 108 . 